\title{
PHYSICAL, PSYCHOLOGICAL AND VIRTUAL REALITIES ${ }^{1}$
}

\author{
Max Velmans, Department of Psychology, Goldsmiths, University of London, New Cross SE14 6NW
}

In J. Wood (ed) The Virtual Embodied. London: Routledge, 1998, pp45-60

\section{Introduction}

In our predominantly materialist culture we take it for granted that the physical world is real. But in what sense are experiences, thoughts and feelings real? And what about virtual realities? Are they physical, psychological, or somewhere in between? In the present chapter I examine:

1. the ways physical, psychological and virtual realities differ

2. the ways in which physical, psychological and virtual realities are the same or 'thing-itself'.

3. the ways in which physical, psychological and virtual realities might relate to some 'grounding reality'

I also tell a story about the possible consequences of living too long in a convincing, virtual reality. typical beliefs about physical, psychological and virtual realities

In our everyday intuitions it is common to think about physical, psychological and virtual realities in the ways summarised in Table 1.

Table 1. typical beliefs about physical, psychological and virtual realities

physical reality

extended in space - in the world

exists independently of the observer

has tangible properties, e.g. mass and solidity

virtual reality

appears to have extension in space, but has no actual extension

appears to be in the world, but is actually in the mind

existence depends on the interaction of the observer with VR equipment

can appear to have tangible properties (with suitable equipment) but does not have such

properties

\section{psychological reality}

nonextended in space - in the mind existence depends on the observer is relatively intangible and insubstantial

Following Descartes' classical body/mind split into res extensa (stuff that extends in space) and res cogitans (thinking stuff), it is common to think of the physical world as having both extension and location in space. By contrast, psychological realities do not have spatial dimensions, and their location is only metaphorically 'in the mind'. In everyday life (and in classical physics) we take it for granted that the physical world continues to exist whether or not we observe it, but psychological realities are only real for a given observer- pains, thoughts and other experiences do not exist in themselves. These intuitions are confirmed by the fact that physical realities have tangible, substantial properties such as mass, solidity and weight. Psychological properties are, by comparison, intangible and insubstantial.

Virtual realities, however, appear to form a third, distinct category. With appropriate headsets, feedback from bodily movements and visual displays they may give the appearance of being virtual worlds extended in physical space, but they have no actual 3D physical extension. One may appear to move around such virtual worlds, but these apparent changes in self-location do not correspond to actual changes in location. Such virtual worlds are 'physical' in so far as their existence depends on the information provided by appropriate physical equipment, but unless this information is translated into an observer's experience, no independent 'virtual reality' exists. In principle, virtual objects can be given what appear to be physical properties, for example, the observer may wear a gauntlet which is programmed to resist closing around a visually perceived, virtual object, making the latter feel 'solid'. In truth, however, there is nothing solid there.

\section{what is taken for granted}

These intuitions about physical, psychological and virtual realities are grounded in widespread assumptions about how minds relate to bodies, and about how perception works. For example in the 'dualist model' shown in Figure 1, light reflected from an external object (a cat) innervates the visual system of a subject to produce 
representations of the cat in the brain. Under appropriate conditions, neural causes and correlates of a conscious experience are formed, accompanied by a percept of a cat 'in the mind' of the subject. The external cat (in the world) and the subject's visual system and brain are 'physical', although they are all clearly separate 'bits' of the physical world. The percept of a cat is even further separated from the external cat, being 'in the subject's mind' (without location and extension in space).

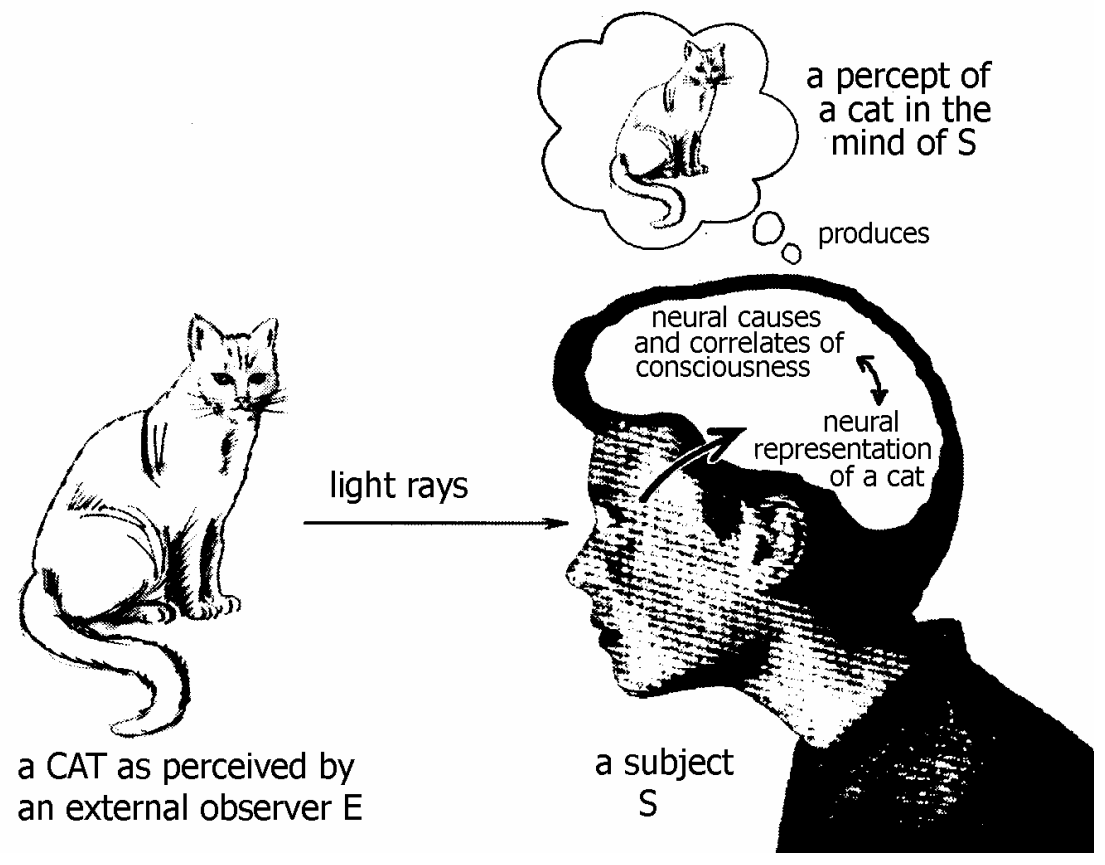

Figure 1. A dualist model of perception

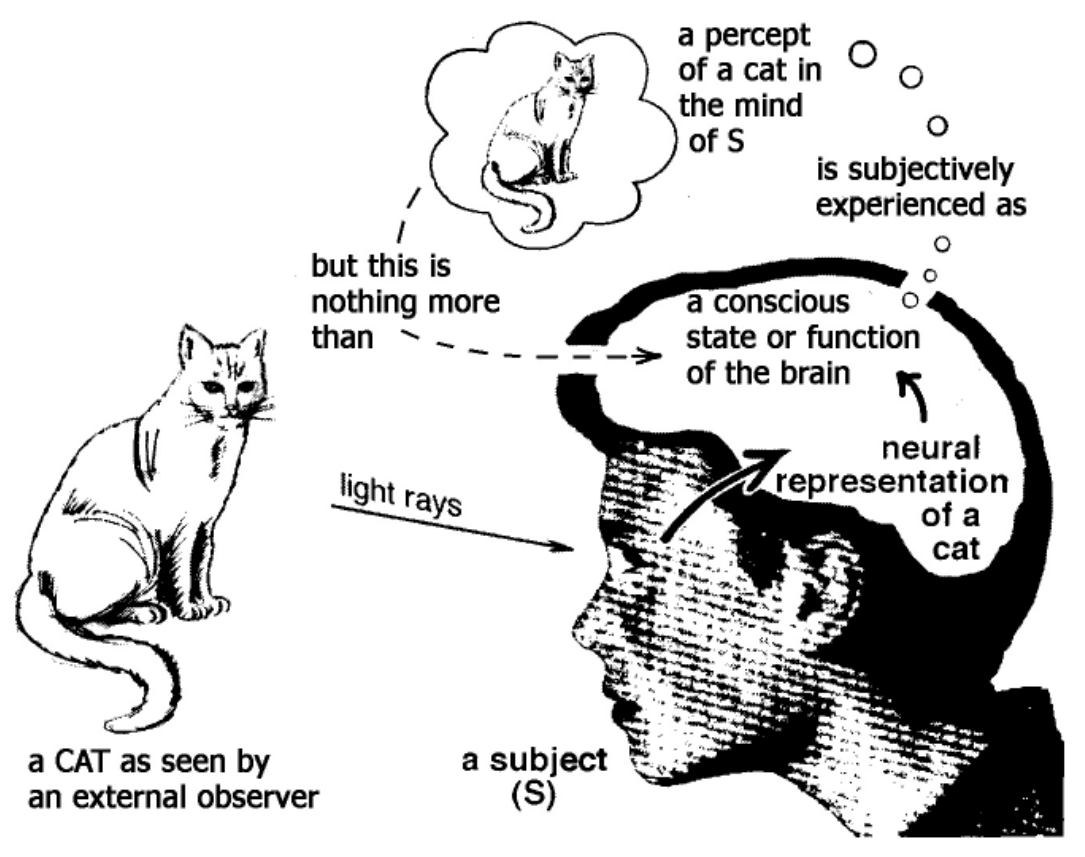

Figure 2. A reductionist model of perception

The 'reductionist model' shown in Figure 2 is very similar. As before, the external cat reflects light rays which innervate the visual system. As before, neural representations of the cat are formed, along with the neural causes and correlates of consciousness. However, reductionists try to demonstrate that the resulting experience (the percept of a cat) is nothing more than a state or function of the subject's brain.

In their effects on our everyday intuitions about physical, psychological and virtual realities, the differences between dualist and reductionist models are not as important as what they shore. Both models take it for granted that the cat as-perceived in the world is quite separate from the percept of a cat. Their dispute is largely about 
whether the percept of a cat is 'in the mind' or 'in he brain'

\section{fitting virtual reality into a model of perception}

Note that virtual realities do not fit easily into such models of the world as-perceived. Dualists and reductionists assume that experiences either have no location or extension, or are located and extended in the brain. However, in VR one appears to interact with a virtual world outside one's body although there is no actual (corresponding) world there. That is, the VR world appears to have 3D location and extension outside one's body in spite of the fact that it is entirely a phenomenal experience. The VR does not seem to be without location or extension, or to be 'in the brain'.

To accommodate virtual reality one needs a model of perception that more accurately portrays the phenomenology of what is experienced. Such a 'reflexive model' of perception' is shown in Figure 3.

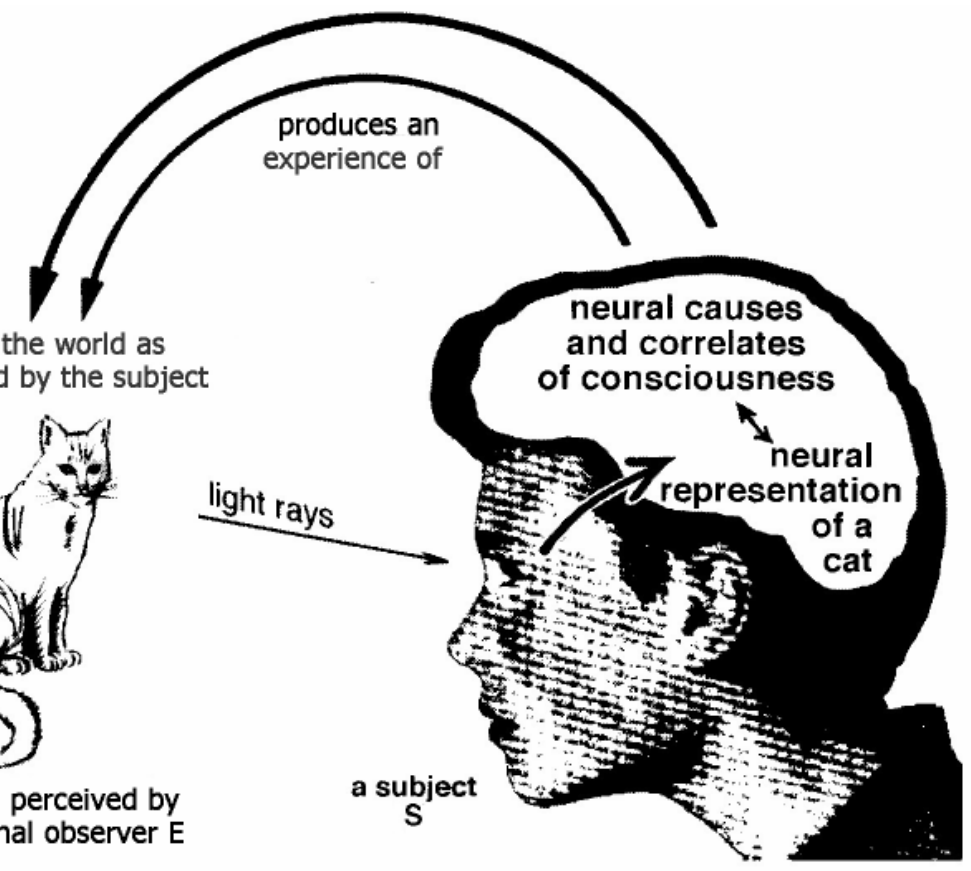

Figure 3. A reflexive model of perception

In most respects, there is no difference between the dualist, reductionist and reflexive models. All the models assume there to be an initiating stimulus (in this case, a physical cat), reflected light, innervation of the visual system, and the formation of neural representations along with neural causes and correlates of conscious experience in the subject's brain. The models differ only in the way they characterise the resulting experience. In the dualist model, the subject's experience of a cat is separate from the physical universe, without location or extension in space. In the reductionist model, the subject's experience of a cat is nothing more than a state or function of the brain. In the reflexive model, the subject's experience of a cat is just the cat as perceived out in space. That is, an entity in space, once it engages the visual system, is experienced as an entity in space. That is why the entire process is 'reflexive' (see Velmans, 1990, 1993, 1996 for a more detailed account).

Only the last claim conforms to our actual experience. Note too, that while we focus on the cat, a cat in the world is all we experience. The neural causes and correlates of what we experience are in the brain - but, subjectively, we have no additional 'experience of a cat' in the mind or brain. Nor can such an experience be found in the brain if it is inspected from the outside, by an external observer. That is, the 'experience of a cat' (in the mind or brain) portrayed in dualist and reductionist models is a theoretical fiction. Applying 'Occam's Razor', the reflexive model gets rid of it, giving a description of what is going on that reflects actual phenomenology.'

\section{how can experiences be located and extended in space?}

Like other models of perception, the reflexive model takes it for granted that the neural causes and correlates of experience are in the brain. Given this, how could the resulting experiences be anywhere else? This is an important question. But it is important to distinguish descriptions of what happens from investigations of how it happens. Before investigating a phenomenon it is necessary to notice it and name it. The reflexive model describes what happens. Perceptual processing in the brain can result in experiences that have a subjective location and extension beyond the brain. In Velmans' I have called this phenomenon 'perceptual projection'. How spatial encodings and other encodings in the brain are translated into such spatial phenomenology are matters for scientific research. 


\section{the evidence for perceptual projection}

There is extensive evidence for perceptual projection in all sense modalities (in truth, very little of what we experience gives the appearance of being located in the brain). I have reviewed this in Velmans (1990) so in what follows I will give just a few examples of the ways in which neural activity (in the brain) can result in spatially located, extended experiences.

\section{projected pain}

Suppose you stab your finger with a pin. You will experience pain. The damage to the skin is 'physical'. By contrast, philosophers of mind universally agree that the pain that results is an 'experience'. In this situation, the physical damage is located in the finger. But where is the pain? Is the pain 'in the mind', or 'nowhere' as dualists claim? Is it literally 'in the brain' as reductionists claim? Or is it in the finger as the reflexive model claims (in the region of damage to the skin)? If you have any doubts about this, why not try it for yourself?

Note that within a dualist or reductionist world view it does not make sense to describe an experience as being 'in the finger'. Conversely, if the pain really is in the finger, there must be something wrong with the way dualists and reductionists describe experience. There are, of course, neural fibers which convey pain signals to the brain located in the finger - so even if one accepts that the pain is in the finger, perhaps one could still regard these neural fibers as an extension of the brain. However, if the arm itself is severed from the shoulder one can still experience pain in a now non-existent 'phantom limb' or 'phantom hand', along with sweating, itching and so on (Sherman, 1996). The brain combines information arriving from the stump with information in memory to produce a hallucinatory arm and hand extended in space - a clear case of 'perceptual projection'.

\section{projected tactile sensations}

Given the deep split between mind and body implicit in dualism, it is crucial to note that the ability of the brain to project pain applies equally to properties more often thought of as 'physical' than 'mental'. 'Hardness' and 'solidity', for example, are usually thought of as physical properties. Nevertheless, the hardness and solidity we experience results from the way mechanical deformations of the skin are interpreted by the brain and projected (reflexively) to the location of the stimulated sense organs. Significantly, such perceptual projection to the body surface is applied even to tactile sensations produced by direct stimulation of the brain. Penfield and Rassmussen (1950) for example, found that micro-electrode stimulation of the somatosensory cortex produces feelings of numbness and tingling which are subjectively located in different regions of the body, not in the brain - another clear case of 'perceptual projection'.

\section{projected auditory sensations}

Our cultural splitting of 'physical' from 'mental' events is also customary in the area of audition. Within dualist and reductionist theory, sounds are usually thought of as physical events out in space, which must be distinguished from experiences of sound 'in the mind' or 'in the brain'. However, the auditory sensors do not detect sounds as such. Rather, they pick up patterns of pressure variation in the air, which produce vibrations at the eardrums, whose intensity, frequency and phase relationships are neurally encoded by hair-cells in the inner ear, and conveyed via the auditory nerve to the brain. But there is no 'experience of sound' in the brain. The experienced sound which results from such stimulation is projected by the brain to the judged location of the acoustic stimulus, resulting in a sound as-perceived out in space.

Manipulations of sound localisation, furthermore, provide a particularly clear example of how perceptual projection can operate in different ways to produce an experience that can be subjectively located either 'in the head' or 'out in space' (thereby undermining this conventional way of distinguishing what is 'physical' from what is 'experience'). That is, subjective location and extension can change although, in other respects, the experienced sounds are the same (they have the same 'qualia' or experienced characteristics). For example, a symphony orchestra played through stereo speakers appears to be distributed in the space outside one's body. But if the same music is played through stereo headphones, the instruments appear to be distributed around the space inside one's head.

Note how difficult it is to maintain any phenomenal distinction between sounds as-perceived and percepts of sound in this last situation. While the orchestral music is subjectively located inside one's head, it seems absurd to claim that there is some additional perception of the music 'inside one's mind or brain'. There are neural causes and correlates of this auditory experience, but phenomenologically, the music as-perceived (inside one's head) and the perception of music are one and the same! Equally, if one switches back from headphones to stereo speakers, it seems absurd to propose that an additional conscious percept of music appears at the precise moment that the music as-perceived switches from being in the head to being out in the world. Nor does it seem plausible to argue that the music as-perceived is transformed from being an 'experience' to being something 'physical' as it moves from being in the head to being part of the world as-perceived outside one's body, for apart from its changed location, it undergoes no other change in its 'qualia'. 
Studies of 'inside the head locatedness' suggest a far simpler explanation. Laws (1972) for example, investigated the acoustic differences between white noise presented through headphones (perceived to be inside the head) and white noise presented through a speaker at a distance of 3 metres (perceived to be out in the world) using probe microphones positioned at the entrance to the auditory canal. This revealed spectral differences (produced largely by the pinnae of the ear) between the white noise presented through the speaker and white noise presented through the headphones. Ingeniously, Laws then constructed an electrical 'equalising' circuit to simulate these spectral differences and inserted this into the headphone circuit. With the headphones 'unequalised', white noise appears to be inside the head irrespective of loudness. With the headphones 'equalised', the white noise not only appears outside the head but actually recedes as the loudness is decreased (a finding that is of potential use in VR design).

Again, it can hardly be claimed that the insertion of an 'equalising' circuit suffices to convert an 'experience' to something 'physical'. Rather, the experiment establishes that spectral distortions produced by the pinnae (or their absence) inform the brain whether or not the source of sound lies beyond the pinnae (see Blauert, 1983, for a review). The experiential model of the source produced by the brain, i.e. the sound as-perceived, is correspondingly located in the head or beyond the pinnae. Although for different purposes we may refer to the sound being 'physical' or an 'experience' (see note 1), the 'qualia' of the sound, other than its experienced location, do not change. As the Reflexive Model maintains, in terms of phenomenology, a sound as-perceived and a percept of a sound are one and the same.

\section{perceptual projection in vision}

As we are visually dominant creatures, the contrast between physical, psychological and virtual realities in the visual modality is of particular interest - for it is in this modality, more than any other, that the apparent separation of the external world as-perceived from what is 'in the mind' seems most clear. For example, visually perceived objects extended in the three-dimensional space around our bodies seem to have very different qualities to visual images of those objects. If visual images exemplify the 'contents of consciousness', then how could objects asseen do likewise?

The reflexive model does not seek to minimise these differences in how objects and images are experienced, for in all probability, they represent discontinuities that from the point of view of human interaction with the world, are as important as they are real. Nevertheless, the fact that phenomenal objects are experienced to be different from images does not alter the fact that both phenomenal objects and images are experienced - that they are equally, in a sense, dependent on perceptual processing in the brain.

Now the dependence of visual imagery on cerebral processing is widely accepted - it is consonant, after all, with the conventional assumption that images are 'in the mind'. By contrast, the very spatial separation of objects asperceived from brains as-perceived makes their interdependence more difficult to imagine. Nevertheless, the evidence for cerebral involvement in the 'construction' of objects as-seen, including their seen location in three dimensional space, is compelling. It is well known, for example, that as an object recedes, its perceived size decreases far less than its optical projection on the retina would suggest (the phenomenon of 'size constancy'). That is, perceived size varies not only with the projected retinal image but also with judged distance - and the judged distance of an object is itself influenced by interpretative cerebral processes operating on retinal size, binocular disparity, ocular convergence, textural gradients, the interposition of other objects, motion parallax and so on.

Indeed, three-dimensional phenomenal space can itself be shown to be, at least in part, a 'construct' of the brain. Common examples of such constructive processes are the experiences of three-dimensionality produced by visual cues suitably arranged on a two-dimensional surface, as in stereoscopes, stereoscopic pictures (which develop into three-dimensional scenes if one looks through them rather than at them), and holograms. For centuries, artists have achieved similar effects by the use of aerial perspective, linear perspective, gradients of size and texture, and so on. (See Peter Cresswell's Chapter 8 for a survey of some of these methods.) As Shepard (1983) points out, the perception of depth produced in paintings is normally muted by the counteracting information provided by binocular disparity, and by the two-dimensional surround which tells us that we are in fact looking at a two-dimensional surface. However, try inspecting Figure 4, a painting by Peter Cresswell, through a rolled-up tube of paper, so as to hide the picture edge. In spite of the use of monocular vision, a strong threedimensional effect should result! 


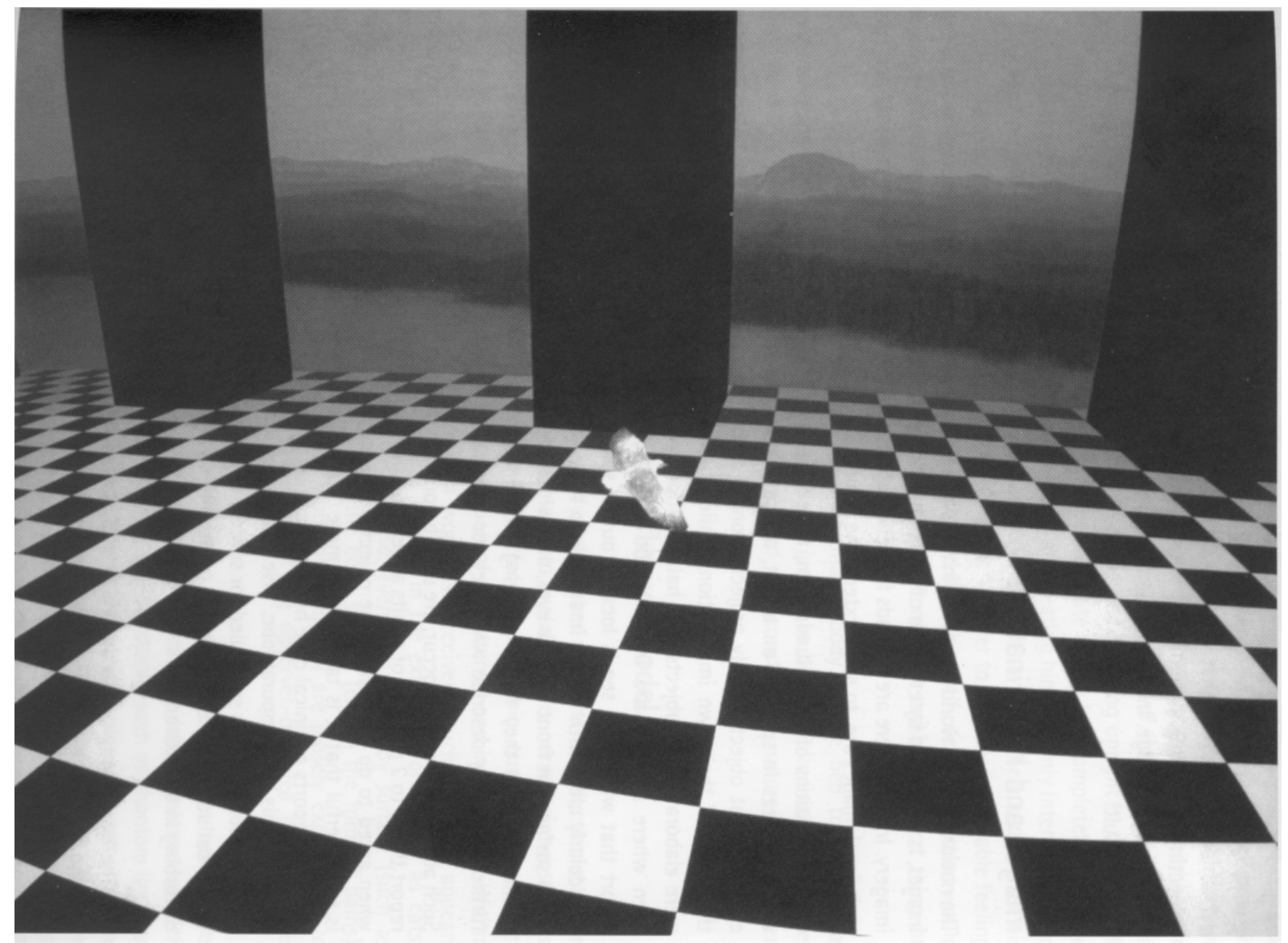

Figure 4. A painting by Peter Cresswell using radial perspective

\section{images and hallucinations}

The notion that the world as-seen is in its own way as much a construct of the brain as are visual images, is supported by evidence of functional similarities between visual perception and visual imagery. Indeed, there are grounds for believing that the processes that produce visual percepts and visual images are, to some extent, the same.

Under conditions of poor illumination, for example, it can be very difficult to decide whether what one sees in space is an object, a visual image or an hallucination. Perky (1910) found, for example, that objects faintly projected on to a screen were often judged by subjects to be the result of their own imagination, while Kulpe (1902) found that dimly lit, fluctuating stimuli were elaborated by subjects into hallucinations which they, nevertheless, judged to be 'real'. Even where subjects are in no doubt that they are imaging or hallucinating, they may report that what they see is located out in space (as opposed to 'inside the head'). This is particularly clear with eidetic imagers who typically report their visual images to be projected on to surfaces in front of their eyes, and which seem to them to be quite distinct from visual memories which they report as being 'inside their head'. When they describe such images, furthermore, they describe what they see as opposed to what they have seen (Haber, 1979).

Such abilities, when they occur, are usually found in children. However, Spanos, Ham and Barber (1973) report that 1 to 2 per cent of adults appear to have the ability to hallucinate an object in a room when asked to do so without the object being present." Very occasionally, the hallucination is so powerful that it is taken to be more 'real' than that which actually exists. Brugger (1994), for example, reports a clinical case history of a young man of 17 suffering from epilepsy caused by a lesion in his left temporal lobe:

" He was being treated with anti-convulsant drugs to control the condition and was scheduled for surgery when he experienced an 'heautoscopic' episode (a visual hallucination of his body combined with an out-of-body experience) which was disturbing in the extreme: The heautoscopic episode, which is of special interest to the topic of this report, occurred shortly before admission. The patient stopped his phenytoin medication, drank several glasses of beer, stayed in bed the whole of the next day, and in the evening he was found mumbling and confused below an almost completely destroyed large bush just under the window of his room on the third floor. At the local hospital, thoracic and pelvic contusions were noted ... The patient gave the following account of the episode: on the respective morning he got up with a dizzy feeling. Turning around, he found himself still lying in bed. He became angry about 'this guy who I knew was myself and who would not get up and thus risked being late for work'. He tried to wake the body in bed first by shouting at it; then by trying to shake it and then repeatedly 
jumping on his alter ego in the bed. The lying body showed no reaction. Only then did the patient begin to be puzzled about his double existence and become more and more scared by the fact that he could no longer tell which of the two he really was. Several times his body awareness switched from the one standing upright to the one still lying in bed; when in the lying in bed mode he felt quite awake but completely paralysed and scared by the figure of himself bending over and beating him. His only intention was to become one person again and, looking out of the window (from where he could still see his body lying in bed), he suddenly decided to jump out 'in order to stop the intolerable feeling of being divided in two'. At the same time, he hoped that 'this really desperate action would frighten the one in bed and thus urge him to merge with me again'. The next thing he remembers is waking up in pain in the hospital." (Ibid., pp. 838-839)

In short, this patient mistakenly judged the hallucinated body on the bed to be his real one and tried to get rid of his real body (which he judged to be the hallucination) in order to become unified again - a powerful example of the constructed nature of embodied experience.

\section{how virtual realities fit into the reflexive model}

As noted above, virtual realities do not fit easily into either a dualist or reductionist vision of the world. But in the reflexive model they are easy to explain. Under normal circumstances, information detected by the sense organs on the surface of, or within, our bodies is subject to perceptual processing to produce a representation of our external and inner worlds. Within subjective experience this takes the form of a world as-perceived, much of which appears to be extended and located in space. Entities located in the external world (such as cats) are usually experienced to be in the external world, events on the body surface (such as skin damage) are usually experienced to be on the body surface (e.g. in the form of pains), and events originating in the mind or brain (such as thoughts) are loosely experienced to be in the mind or brain. In each case the result of the observer-observed interaction is 'reflexive'.

These biologically determined constructions normally represent the energies and events surrounding and within our bodies well enough to support successful, adaptive interaction with the world. However, misrepresentation is also possible. Hallucinations such as phantom limbs or heautoscopic experiences are cases where 'inner' information is mistakenly projected on to the external world. In the manner shown in Figure 3.6, virtual reality systems artificially engage the same normal, constructive processes (including perceptual projection) to produce experienced, artificial worlds. In so far as the information input from VR systems is similar to the 3D information supplied by the normal world, they will be experienced as facsimiles of the normal world located and extended in 3D space.

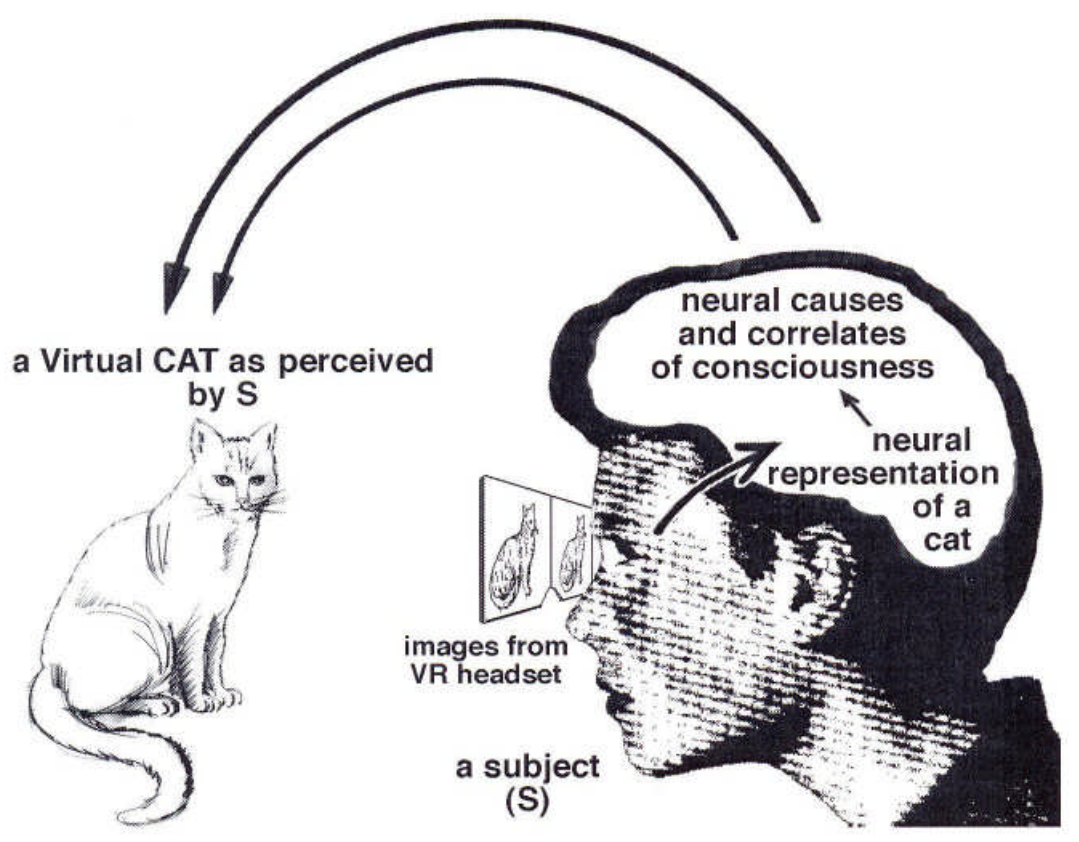

Figure 5. A virtual model of perception

\section{physical, psychological and virtual realities}

Within the reflexive model the physical world as-experienced is part of the contents of consciousness. The contents of consciousness are not in some separate place or space 'in the mind' or 'in the brain'. That is, no phenomenal distinction can be drawn between what we normally think of as the 'physical world' and the 'world as- 
experienced'. With our eyes open the 'physical world' is what we experience. This provides a completely different view of the mind/body relationship (discussed in more depth in Velmans 1990, 1993, 1996). Suffice it to say that once experienced physical reality is included within this extended view of psychological reality it no longer makes sense to split experienced physical reality from psychological reality (in the ways shown in Table 1). Some aspects of psychological reality (such as thoughts) have no clear phenomenological extension or location in space and appear to be relatively insubstantial. However, much of psychological reality (the experienced external world) does seem to have spatial location and extension and is experienced to have substantial properties such as hardness and weight. The way such properties are experienced depends on the perceptual systems of the observer, as well as on the properties of the observed. The phenomenal world constructed by the mind or brain from information detected by the sense organs is, at once, psychological and physical.

Note, however, that this experienced 'physical world' may be very different from the world described by Physics, for example, in terms of Relativity Theory or in terms of Quantum Mechanics. That is, the experienced 'physical world' is just one, biologically determined, representation of energies and events surrounding and within our bodies which Physics, Chemistry, Biology, Psychology and other sciences might represent in very different ways. Within the reflexive model these are alternative representations of some underlying reality or 'thing-itself'. The relative utility of these representations can only be judged in terms of the purposes for which they are to be used.

Virtual realities are artificial worlds as-experienced. Like the everyday world that we take to be 'real', they are phenomenal models constructed by the mind or brain, in this case of the information fed to the sense organs by VR equipment. This dependence on VR equipment loosens the constraints of the natural world. Some VR systems might nevertheless provide a facsimile of the natural world; examples include flight simulators, and systems that provide training for surgeons in a virtual operating theatre. Other VR systems produce experienced worlds that are entirely fantastic or fanciful.

The human ability to represent actual states of affairs along with the ability to image or dream hypothetical or imaginary worlds is as old as human history; much intellectual endeavour, now as then, is devoted to the need to distinguish the one from the other. VR systems extend the domain of our imagination, blurring the boundaries between what is imaginary and what is real. Their impact on human life will depend on how convincing they become.

\section{peering into the crystal ball}

It is notoriously difficult to predict how far new technologies will develop or what their social impact will be. However, let us suppose that VR worlds eventually become so convincing that they are no longer clearly distinguishable from actual worlds (this blurring of imagination into reality already exists, for some, in vivid dreams). This would relativise what we normally take to be 'reality': that is, the everyday 'physical world' asperceived would be seen as one (biologically given) construction out of many possible constructions. For some this might be threatening. In the development of Western thought, human ethnocentricity has already been shaken by the revelations that the Earth is not the centre of the Universe (Copernicus); that man is not the sole focus of creation (Darwin); and that the conscious ego might in some respects be driven by a dynamic unconscious self (Freud) (cf. Tarnas, 1993, p. 422). The relativisation of experienced reality might remove the last prop of our ethnocentric assumptions, adding impetus to the historic search for a firmer, more universal ground.

The ability to engage in unlimited, seemingly real, imaginary worlds might also present real dangers to those who are immersed in them - the most obvious one being a loss in the ability to distinguish what is imaginary from what is real. That is, 'VR junkies' may begin to lose contact with the actual world in which they are embodied, and lose interest in the deepening exploration of its nature, or of their own nature.Engaging in a world of surrogate relationships in cyberspace has similar potential and dangers.

On the one hand one might practice engaging in relationships without the risks attending actual relationships. On the other hand one might engage in virtual relationships as a way of avoiding actual relationships, again losing the possibility of a deepening exploration of their nature.

\section{Janet's dream}

I do not usually tell personal stories in theoretical papers. However, two days after being invited to give this paper to the Embodied Knowledge and Virtual Space Conference, an event occurred which bears directly on its theme. I have decided to tell it as it was.

I had not mentioned anything about the conference to my partner Janet. But that night (about four in the morning) I was suddenly woken by a shout. Janet was sitting up in bed, clearly disoriented. She had been dreaming about being enclosed in a bubble. I asked her what the bubble meant. After some thought she said it had something to do with virtual reality. Given that I had not mentioned VR to her, this seemed odd - so we switched on the light and talked about her dream. 


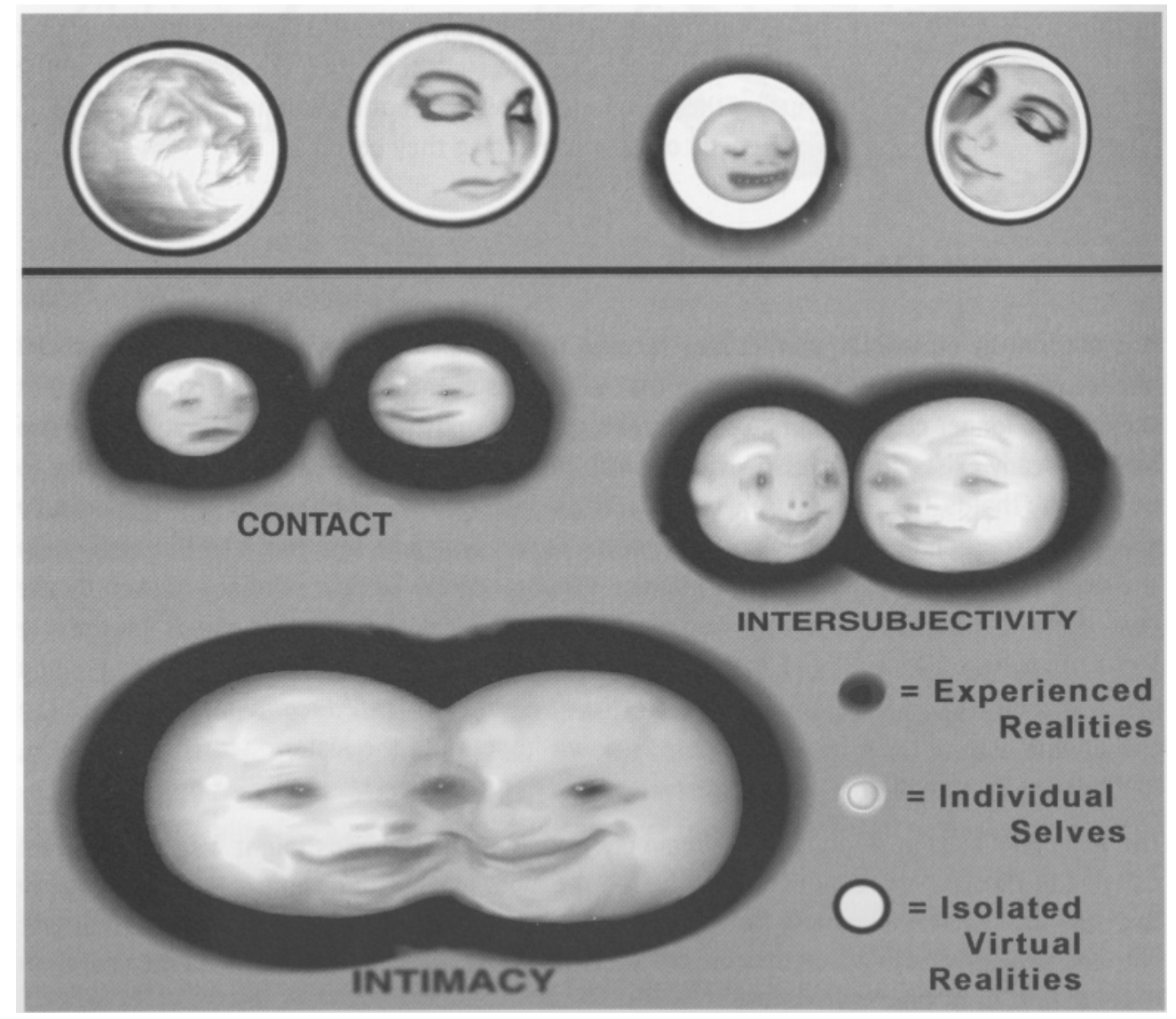

\section{Figure 6. Relationships between individual selves and virtual realities}

In the dream there were many people enclosed in bubbles. These bubbles were virtual worlds existing entirely 'in people's minds'. In their own way these were absorbing and beguiling. While people remained in these separate, virtual worlds they might engage in virtual relationships, but there was no genuine contact between them. They remained in their own individual bubbles. In 'real' life people were also in bubbles. But for these people, a genuine engagement with embodied being and an openness to life made their bubbles semi-permeable. Here there was the possibility of genuine exploration with others. Being semi-permeable, these bubbles allowed for contact, intersubjectivity and, sometimes, for genuine intimacy (see Figure 6).

To begin with, people had a choice about whether or not to enter into virtual reality or to engage in embodied life. But, gradually, people began to separate into two distinct streams. As the virtual worlds became more convincing, those who chose to spend most of their time in them began to seal themselves off from embodied life, and to lose the ability to distinguish the virtual from the real. Eventually, they became lost in endless games. By contrast, people who chose to explore the potential of embodied being and to remain exposed to the 'realities' of life gradually expanded their experience to become more deeply grounded in their own nature.

In her dream, Janet found herself in a 'virtual bubble' in the wrong stream. With a shout she burst out of the bubble to join the other stream. Make of it what you will.

\section{References}

Blauert, J., Spatial Hearing: the Psychophysics of Human Sound Localization, Cambridge, Mass., MIT Press, 1983.

Brugger, P., 'Heautoscopy, epilepsy, and suicide', Journal of Neurology, Neurosurgery, and Psychiatry, 57, 1994, pp. 838-839.

Haber, R.N., 'Twenty years of haunting eidetic imagery: where's the ghost?', Behavioral and Brain Sciences, 2, 1979, pp. 583-619.

Kulpe, 0., 'Uber die Objectivirung und Subjectivirung von Sinneseindruken', Philosophische Studien, 19, 1902, pp. 508-536.

Laws, P., 'On the problem of distance hearing and the localization of auditory events inside the head', Dissertation, Technische Hochschule, Aachen. Cited in Blauert, J., Spatial Hearing: the Psychophysics of Human Sound Localization, Cambridge, Mass., MIT Press, [1972], 1983.

Penfield, W. and Rassmussen,T.B., The Cerebral Cortex of Man, Princeton, NJ, Princeton University Press, 1950. Perky, C.W., 'An experimental study of imagination', American Journal of Psychology, 21, 1910, pp. 422-452. Shepard, R.N., 'Ecological constraints on internal representation: Resonant kinematics of perceiving, imagining, 
thinking, and dreaming', third James J. Gibson Memorial Lecture given at Cornell University, 2 October 1983. Sherman, R.A., Phantom Pain, New York, Plenum Publishing Corporation, 1996.

Spanos, N.P., Ham, M.H. and Barber, TX, 'Suggested ("hypnotic") visual hallucinations: experimental and phenomenological data', Journal of Abnormal Psychology, 81, 1973, pp. 96-106.

Tarnas, R., The Passion of the Western Mind, New York, Ballantyne Books, 1993.

Velmans, M., 'Consciousness, brain, and the physical world', Philosophical Psychology, 3,1990, pp. 77-99. Velmans, M., 'A reflexive science of consciousness', in Experimental and Theoretical Studies of Consciousness, CIBA Foundation Symposium 174, Chichester, Wiley, 1993, pp. 81 -99.

Velmans, M., 'What and where are conscious experiences?', in Velmans, M. (ed.), The Science of Consciousness: Psychological, Neuropsychological and Clinical Reviews, London, Routledge, 1996.

\footnotetext{
${ }^{1}$ The web version of this chapter is formatted slightly differently from the original and Figures 1, 2 and 3 have been updated. Otherwise the chapter is the same.

2 In the reflexive model the 'cat as-experienced' is phenomenologically identical to one's 'experience of the cat'. However, these referring expressions are somewhat different in meaning. All experiences result from an interaction of observed events and energies with the perceptual apparatus of an observer. These different expressions direct our attention to either the observed or to the observer. When we refer to the 'cat' or to the 'cat as-experienced', we are usually more interested in the nature of the observed. When we refer to an 'experience of the cat' we are usually more interested in the involvement of the observer. This does not alter the fact that only one experience results from this observer-observed interaction - a phenomenal cat out in the world.
} 